\title{
A Síndrome de Burnout em professores do Ensino Médio da Cidade de Exu - PE
}

\author{
Burnout Syndrome in High School teachers in the City of Exu - PE \\ Síndrome de Burnout en docentes de Secundaria de la Ciudad de Exu - PE
}

Leonardo Eric Silva Santos

ORCID: https://orcid.org/0000-0002-5850-1911

Universidade Regional do Cariri, Brasil

E-mail: leonardoeric22@yahoo.com

Dennis Bezerra Correia

ORCID: https://orcid.org/0000-0002-7782-4767 Universidade Regional do Cariri, Brasil

E-mail: denniscorreia40@gmail.com

Cícero Jorge Verçosa

ORCID: https://orcid.org/0000-0002-3284-6719 Secretaria de Educação e Esportes de Pernambuco, Brasil

E-mail: cjvercosa@hotmail.com

Jailson Renato de Lima Silva

ORCID: https://orcid.org/0000-0003-1292-8060 Universidade Regional do Cariri, Brasil

E-mail: jailsonslrj@outlook.com

Rafael Henrique Luciano dos Santos

ORCID: https://orcid.org/0000-0001-6968-6803 Secretaria de Educação e Esportes de Pernambuco, Brasil E-mail: rafaelhenrique.bio@gmail.com

Maria Eliana Vieira Figueroa

ORCID: https://orcid.org/0000-0002-0049-4456 Secretaria de Educação e Esportes de Pernambuco, Brasil E-mail: elianavfigueroa1@gmail.com

Clêidio da Paz Cabral

ORCID: https://orcid.org/0000-0003-1825-4925 Secretaria de Educação e Esportes de Pernambuco, Brasil

E-mail: cabralbahia@hotmail.com Francileide Vieira Figueirêdo

ORCID: https://orcid.org/0000-0002-6853-854X Universidade Regional do Cariri, Brasil E-mail: francileidevf@gmail.com

João Paulo Camilo de Oliveira

ORCID: https://orcid.org/0000-0003-0286-1149 Universidade Regional do Cariri, Brasil

E-mail: camilodeoliveirajoaopaulo35@ gmail.com

Ginna Gonçalves Pereira

ORCID: https://orcid.org/0000-0003-0267-2615 Universidade Regional do Cariri, Brasil E-mail: ginna.pereira@urca.br

Maria Magnolia Batista Florêncio

ORCID: https://orcid.org/0000-0003-1919-1524 Secretaria de Educação e Esportes de Pernambuco, Brasil

E-mail: magnoliabf1@gmail.com

Allyson Francisco dos Santos

ORCID: https://orcid.org/0000-0001-8635-6398 Secretaria de Educação e Esportes de Pernambuco, Brasil E-mail: allysons@outlook.com.br

Jose Aglailson Oliveira da Anunciação

ORCID: https://orcid.org/0000-0003-1751-2953

Universidade Regional do Cariri, Brasil E-mail: aglailsonoliveira@gmail.com

Nayane Kely Silva Sampaio

ORCID: https://orcid.org/0000-0001-6310-5529 Universidade Regional do Cariri, Brasil E-mail: nayane-kelly@ hotmail.com

Gabriel Venancio Cruz

ORCID: https://orcid.org/0000-0002-0006-5213 Universidade Regional do Cariri, Brasil

E-mail: gabrielvenancio02@ hotmail.com 


\author{
Francisco Diego Pereira \\ ORCID: https://orcid.org/0000-0002-4816-3381 \\ Universidade Regional do Cariri, Brasil \\ E-mail: diego.vilar.pereira@gmail.com \\ Josefa Edilene da Silva \\ ORCID: https://orcid.org/0000-0001-8635-6398 \\ Universidade Regional do Cariri, Brasil \\ E-mail: Edilene-calixto1@ @otmail.com \\ Filipe Gutierre Carvalho de Lima Bessa \\ ORCID: https://orcid.org/0000-0001-8126-8831 \\ Universidade Estadual Vale do Acaraú, Brasil \\ E-mail: filipe_carvalho@uvanet.br \\ Elane Soares Araújo \\ ORCID: https://orcid.org/0000-0003-1690-9406 \\ Universidade Regional do Cariri, Brasil \\ E-mail: elane802@gmail.com \\ Cicero Magerbio Gomes Torres \\ ORCID: https://orcid.org/0000-0002-3585-452X \\ Universidade Regional do Cariri, Brasil \\ E-mail: cicero.torres@urca.br \\ Norma Suely Ramos Freire \\ ORCID: https://orcid.org/0000-0002-1556-9377 \\ Universidade Regional do Cariri, Brasil \\ E-mail: normarfb@hotmail.com
}

\title{
Resumo
}

A Síndrome de Burnout é uma patologia que afeta profissionais de muitas áreas, em especial professores. A exaustão e despersonalização são um dos sintomas que geralmente irão estar presentes na pessoa acometida. Esta pesquisa objetivou analisar sinais e sintomas da síndrome de Burnout em professores de ensino médio, que atuam no programa de tempo integral. Trata-se de um estudo descritivo com abordagem quantitativa que é definido como o levantamento sistemático dos problemas, características ou fenômenos observados na população. O estudo foi desenvolvido em uma escola de ensino médio da cidade de Exu que funciona na modalidade de ensino de tempo semi-integral. A fim de preservar a identidade da escola o seu nome não foi indicado no estudo. A amostra da pesquisa constitui de 9 professores. A pesquisa utilizou-se da aplicação em forma presencial de questionários estruturados contendo quatro blocos de perguntas. Diante do exposto, é imprescindível que essa pesquisa seja ampliada a partir de novas contribuições e faz-se necessário uma reflexão acerca das suas falhas ou limitações, a fim de se definir melhorias para que possibilite o alcance de resultados ainda mais significativos no campo das aprendizagens e do desenvolvimento pessoal. O conhecimento acerca dos fatores preditores da síndrome de Burnout facilita a elaboração de medidas e programas de prevenção. Ressalta-se que este estudo apresenta limitações no que se refere ao número de participantes, uma vez que a amostra não foi atingida em razão da não participação de muitos professores na coleta dos dados.

Palavras-chave: Síndrome de Burnout; Profissional da Educação; Docência; Ensino Médio.

\begin{abstract}
Burnout Syndrome is a pathology that affects professionals in many areas, especially teachers. Exhaustion and depersonalization are one of the symptoms that will usually be present in the affected person. This research aimed to analyze signs and symptoms of Burnout syndrome in high school teachers who work in the full-time program. This is a descriptive study with a quantitative approach that is defined as the systematic survey of problems, characteristics or phenomena observed in the population. The study was carried out in a high school in the city of Exu that works in a semi-full-time teaching modality. In order to preserve the school's identity, its name was not indicated in the study. The research sample consists of 9 teachers. The research used the face-to-face application of structured questionnaires containing four blocks of questions. Given the above, it is essential that this research is expanded from new contributions and it is necessary to reflect on its failures or limitations, in order to define improvements to enable the achievement of even more significant results in the field of learning and personal development. Knowledge about the predictors of Burnout syndrome facilitates the development of prevention measures and programs. It is noteworthy that this study has limitations with regard to the number of participants, since the sample was not reached due to the non-participation of many teachers in data collection.
\end{abstract}

Keywords: Burnout syndrome; Education Professional; Teaching; High school.

\section{Resumen}

El Síndrome de Burnout es una patología que afecta a los profesionales de muchas áreas, especialmente a los docentes. El agotamiento y la despersonalización son uno de los síntomas que suele estar presente en la persona afectada. Esta investigación tuvo como objetivo analizar los signos y síntomas del síndrome de Burnout en profesores de secundaria que trabajan en el programa de tiempo completo. Se trata de un estudio descriptivo con enfoque cuantitativo que se define como el relevamiento sistemático de problemas, características o fenómenos observados en 
la población. El estudio se llevó a cabo en una escuela secundaria de la ciudad de Exu que trabaja en una modalidad de docencia semi-a tiempo completo. Para preservar la identidad de la escuela, su nombre no se indicó en el estudio. La muestra de investigación consta de 9 profesores. La investigación utilizó la aplicación presencial de cuestionarios estructurados que contienen cuatro bloques de preguntas. Dado lo anterior, es fundamental que esta investigación se amplíe a partir de nuevos aportes y es necesario reflexionar sobre sus fallas o limitaciones, a fin de definir mejoras que permitan la consecución de resultados aún más significativos en el ámbito del aprendizaje y el desarrollo personal. El conocimiento sobre los predictores del síndrome de Burnout facilita el desarrollo de medidas y programas de prevención. Es de destacar que este estudio tiene limitaciones en cuanto al número de participantes, ya que no se alcanzó la muestra debido a la no participación de muchos docentes en la recolección de datos.

Palabras clave: Síndrome de Burnout; Profesional de la Educación; Enseñando; Escuela Secundaria.

\section{Introdução}

O trabalho enquanto atividade laboral faz parte do cotidiano da vida dos seres humanos apresentando grande importância para seu desenvolvimento. É através do trabalho que as pessoas se tornam contribuintes da sociedade em que vivem dentro das diversas profissões existentes (Costa \& Scatolin, 2017). O trabalho, ou atividade laboral, proporciona que as pessoas se sintam realizadas em seus afazeres e gozem de bem-estar, realização pessoal/profissional e prestígio proveniente de seu desempenho, contudo, a depender dos fatores que interfiram no desempenho profissional, a atividade laboral longe de produzir realização pode causar quadros patológicos de ordem física e psicológica (Freitas, 2020).

O profissional da educação apresenta-se atualmente como elo entre o conhecimento científico e a orientação para a vida através de princípio e valores pré-estabelecidos nas diretrizes educacionais do sistema de ensino (Pertile, \& Perin, 2020). Sendo mediador de saberes cria laços de companheirismo, amizade e cumplicidade com os educandos apesar da desvalorização injusta desta profissão no Brasil (Souza \& Heinzle, 2020).

Nos dias atuais os professores enfrentam grandes entraves que refletem em sua prática docente, que vão desde dificuldades pedagógicas decorrentes de salas superlotadas, ausência de apoio didático/pedagógico que lhe causa desgastes de ordem psicossomática. O professor além de se vê obrigado a contornar todas as situações antes pontuadas ainda esbarra nas exigências burocráticas do sistema educacional, ou de programas de tempo integral que por vezes os conduzem ao estresse (Koeppe et al, 2020).

O estresse vem se tornando um problema em várias instituições, das mais variadas áreas de trabalho, sendo as instituições de ensino as mais afetadas conforme literatura consultada, pois, nesse espaço se configura atividades que demandam grande esforço, competência, habilidade e responsabilidade social. Dessa forma, nos dias atuais a função docente passou a comungar não só do ato pedagógico, mas também passou a ser responsável pelo desenvolvimento psicossocial dos seus alunos, o que demanda uma possível carga de estresse. O estresse não é uma doença, mas uma tentativa de adaptação ao ambiente que ele está inserido e relacionado ao cotidiano diário experimentado pelo mesmo. Ressalta-se aqui que o ambiente escolar, dependendo de suas características pode fazer com que o estresse apareça (Borba, 2020).

Zamoner et al (2021) ressalta que muitos problemas permeiam a profissão docente e afetam o ser professor. Sendo uma resposta crônica aos fatores estressantes ocupacionais que pode ocasionar o surgimento da Síndrome de Burnout.

Pinto et al (2015) relata que a síndrome de Burnout é um distúrbio psíquico descrito inicialmente em 1974 por Freudenberg, um médico americano cujas principais características destaca-se como o estado de tensão elevado que está inteiramente ligado a condições desgastantes de trabalho, tanto físicas quanto emocionais. A síndrome se mostra presente principalmente em pessoas cujas profissões exige contato direto e intenso com outras pessoas.

Além do estresse do âmbito de trabalho, Fabricio, et al (2020), trata outros fatores externos que também influenciam a presença dessa síndrome como: separação ou perda de ente querido, desemprego, competição no mercado de trabalho, incerteza de um acontecimento etc. Na atualidade a Síndrome de Burnout, segundo Magalhães, et al (2021), é constituída de três dimensões que são: exaustão emocional, despersonalização e baixa realização pessoal no trabalho. 
A história da educação revela que atividades docentes antes eram consideradas nobres e o professor era conhecido pelo saber do conhecimento. Nos dias atuais acontece o contrário, em virtude das políticas públicas mal acompanhadas e apoiadas por parte dos governantes, contribuem para a decadência da profissão e da ocupação docente, repercutindo assim no educador e no aluno (Bauer et al, 2017)

Lima et al, (2020) ressalta que o professor se sente cobrado para efetuar mudanças no âmbito educacional em um tempo muito curto, se sentindo desta forma sobrecarregado de trabalho, além de precisar lidar com turmas numerosas que geralmente incorre em tensões nas relações professor x aluno, lida com excesso de carga horária e escassez de recursos materiais e didáticos. Esse profissional precisa enfrentando todos esses entraves com sorriso no rosto, com comunicação repleta de gentileza sem esquecer de que tem uma vida própria que também precisa ser cuidada.

Buscando respostas para deterioração da saúde de trabalhadores surgiu nos Estados Unido, na década de 1970, a síndrome de Burnout segundo Mota, et al (2017), definida como um esgotamento profissional. A palavra Burnout de origem inglesa significa queimar para fora, o que está relacionado com alterações psicológicas que podem afetar o desempenho no trabalho. Assim, a síndrome de Burnout foi citada pela primeira vez pelo psicanalista Herbert Freundeberg que relacionou a patologia ao estresse que pessoas passavam em seus dia-a-dia no ambiente de trabalho.

Em meio a todos os entraves, o docente tende a procurar caminhos alternativos, construir pontes afim de amenizar o que pode piorar; relacionados a submersão de problemas que estão presentes em salas de aulas e corredores escolares. Condizente com Jesus, et al (2017), com o passar dos anos os sintomas da Síndrome de Burnet irão ficando mais notórios, assim o que importa é programar todo o conteúdo anual e despejá-lo nas aulas afim de cumprir as horas aulas e conteúdos programáticos.

O interesse pela temática da pesquisa sobre Síndrome de Burnout ocorreu depois do acesso a literatura no tema, disponibilizada em material de apoio pedagógico dentro de uma instituição de ensino em oportunidade de trabalhar, através de minicontrato, deparado com situações que parecem tão corriqueiras em nossos dias, mas, tão pouco reconhecidas pelos envolvidos. Nossa curiosidade foi aguçada ao imaginar na saúde de professores que fazem parte do quadro docente de uma escola de referência para o ensino médio com tempo integral.

Considera-se que o estudo proposto possui relevância, por apresentar um tema pouco debatido no curso de formação docente pretendendo discutir e trazer novas pesquisas e debates para pesquisas futuras. Diante do que foi exposto, o presente trabalho tem como objetivo analisar sinais e sintomas da síndrome de Burnout em professores de ensino médio, que atuam no programa de tempo integral para correlacionar atividade profissional com saúde pessoal.

\section{Metodologia}

\section{Tipo de Pesquisa}

Trata-se de um estudo descritivo com abordagem quantitativa que é definido como o levantamento sistemático dos problemas, características ou fenômenos observados na população estudada (Oliveira et al, 2020). O estudo foi desenvolvido em uma escola de ensino médio da cidade de Exu que funciona na modalidade de ensino de tempo semi-integral. A fim de preservar a identidade da escola o seu nome não foi indicado no estudo. A amostra da pesquisa constitui de 9 professores. A pesquisa utilizou-se da aplicação em forma presencial de questionários estruturados contendo quatro blocos de perguntas. Esse estudo teve também como fonte uma tabela informações de atestados médicos que estão descritos todos os atestados dos professores da escola pesquisada no ano de 2018 e suas respectivas classificações internacional de doenças (CID).

De acordo com os aspetos ético legais das pesquisas envolvendo seres humano, os participantes foram devidamente informados sobre a pesquisa, garantindo o anonimato das informações prestadas, sendo o Termo de Consentimento Livre e Esclarecido (TCLE) fornecido e apresentado, previamente, em duas vias. Esse termo foi assinado por extenso ou através de 
impressão datiloscópica pelas pessoas entrevistadas e nele foram esclarecidos os objetivos do estudo e assegurada à confidencialidade dos dados colhidos com a pesquisa, assim como a liberdade de participar ou de declinar da mesma em qualquer etapa. Todo material coletado (entrevistas e anotações) encontra-se depositado no Núcleo de Estudos e Pesquisa em Ensino de Ciências e Biologia da Universidade Regional do Cariri. Os dados foram analisados qualitativamente com o auxílio do programa Microsoft Excel 2016 (Microsoft Corporation ()), considerando todas as informações dos entrevistados válidas para a construção dos resultados.

\section{Resultados e Discussão}

Analisando-se os dados, observou-se que a maioria dos entrevistados são do sexo feminino, sendo apenas dois do sexo masculino. O grupo está a faixa etária entre 26 a 47 anos. Dos nove professores entrevistados; quatro são casados e os outros cinco são solteiros, quanto a situação da trabalhado grande parte dos entrevistados são professores estatuários exceto um que possui minicontrato.

A síndrome de Burnout afeta tanto homens como mulheres, neste estudo a maioria dos participantes são mulheres, e destas 50\% são casadas e com filhos, o que pode influenciar o nível de estresse das mesmas em decorrência de aumento na quantidade de trabalho. Tratando-se da carga horária a maioria dos entrevistados possuem 40hs de carga horária semanal, com exceção de três - um com apenas 30hs e outro com dois vínculos. Os achados deste trabalho se diferem bastante do que foi relatado por Carvalho \& Macêdo (2020) que menciona em seus estudos têm que os professores do sexo masculino são mais expostos que os do sexo feminino.

Dos nove entrevistados apenas dois, além de lecionarem aulas possuem outro emprego. Um fator que também pode contribuir para uma maior carga de pendências é que além de lecionar, desenvolver outro trabalho que não seja em na sala de aula contribui para que haja uma sobrecarga ainda maior de habilidades para serem desenvolvidas.

Com relação a titulação grande parte dos professores, possuem título de especialista, somente uma tem mestrado. Todos os entrevistados tiveram suas férias recentemente no mês de janeiro de 2019. Segundo Oliveira et al (2020), o maior e melhor bem de uma instituição são as pessoas que lá trabalham e elas devem ser cuidadas e assistidas para que possam produzir melhor. O profissional recarrega sua bateria quando tira férias, pois um colaborador demasiadamente fadigado, não consegue desempenhar suas funções com qualidade. Quando o corpo é exigido passa a trabalharem da força mental, assim desestrutura a capacidade de produção e fragilizando a saúde e seu estado emocional.

Quanto ao lazer dois entrevistados relataram que gostam de assistir filmes, dois gostam de dormir, um deles relatou que gosta de sair com os amigos, enquanto outro apresentou que gosta de cuidar de plantas e outro pontuou que gosta de ler e viajar. A maioria dos entrevistados veem esse fator como primordial para colaborar com a carga de estresse. Assim o tempo disponível dos professores é muito curto para si, ou seja, a maior parte do tempo diário do mesmo é dedicado ao fazer pedagógico Silva \& Ribeiro (2020).

Quanto a "exaustão emocional" - que é um estado atingido pela sobrecarga de esforço. Neste caso, não falamos apenas de excessos de trabalho, mas também de assumir conflitos, responsabilidades ou estímulos emocionais ou cognitivos. A exaustão na escola observada é notada ao chegar-se na sala de professores, onde o espaço dos professores é palco de reclamações dos alunos e sobrecargas de trabalhos.

Em relação à "realização profissional" essa realização vem através das satisfações das necessidades de cada indivíduo. Já a "despersonalização profissional” - o transtorno de despersonalização/desrealização é um tipo de transtorno dissociativo que consiste em sentimentos recorrentes ou persistentes de distanciamento do próprio corpo ou processos mentais, geralmente com uma sensação de ser um observador externo da própria vida (despersonalização) ou de estar desconectado de um ambiente (desrealização). Silva \& Ribeiro (2020). 
Para o fator "realização profissional" apresentou-se na pesquisa 6 (seis) professores que expressaram um nível médio e 3(três) baixo índice nesse fator. Sobre a despersonalização profissional 3 (três) professores encontram-se com baixo índice, apenas 1(um) com índice médio e 5 (cinco) com índice alto. Levando-se em consideração esses aspectos, cabe analisar o papel da autoeficácia no trabalho como preditora de variável que trata sobre a saúde/doença do trabalhador, como a síndrome de burnout (Loiola \& Martins, 2019).

Quadro 1 - índices de resultados por dimensões.

\begin{tabular}{|c|c|}
\hline DIMENSÕES & RESULTADOS \\
\hline \multirow[t]{2}{*}{ FATORES PREDITORES } & $\begin{array}{l}\text { Todos concordaram que o fazer pedagógico requer muito tempo e atenção por } \\
\text { parte deles, o que pode influenciar para consequência da síndrome. }\end{array}$ \\
\hline & Média grupal =100\% \\
\hline \multirow[t]{2}{*}{ EXAUSTÃO EMOCIONAL } & $\begin{array}{l}5 \text { alto índice } \\
3 \text { médio índice } \\
\text { 1baixo índice }\end{array}$ \\
\hline & Média grupal $=54 \%$ \\
\hline $\begin{array}{l}\text { REALIZAÇÃO } \\
\text { PROFISSIONAL }\end{array}$ & $\begin{array}{l}6 \text { médio índice } \\
3 \text { baixo índice }\end{array}$ \\
\hline & Média grupal $=63 \%$ \\
\hline $\begin{array}{l}\text { DESPERSONALIZAÇÃO } \\
\text { PROFISSIONAL }\end{array}$ & $\begin{array}{l}3 \text { baixo índice } \\
1 \text { médio índice } \\
5 \text { alto índice }\end{array}$ \\
\hline & Média grupal $=\mathbf{5 4 \%}$ \\
\hline
\end{tabular}

Fonte: Autoria própria (dados da pesquisa).

Quanto aos sintomas somáticos professores relataram que o fator crucial para o estresse e resquícios de burnout é principalmente, em primeiro lugar, o cansaço mental, depois mencionam a falta de tempo pra si, apresentam em seguida que o excesso de trabalho tem acarretado também falhas na memória e concentração. Diante de todos esses fatores o grau de irritabilidade tende a aumentar e o professor muitas vezes é chamado de chato por parte dos alunos, por não conseguir conter o seu estresse (Penteado \& Souza, 2019).

Ao analisar o objeto de estudo verifica-se que professores participantes da pesquisa estão desmotivados em seus trabalhos profissionais e que passam por situações de inquietações que merecem um olhar de pertencimento por parte do sistema educacional. É possível perceber que os professores participantes desta pesquisa se encontram doentes, principalmente no fator emocional, uma doença da alma que pode reverberar para depressão e possíveis transtornos psicológicos e psicossomáticos aos quais são passíveis ao Burnout.

Amparados nas referências do código internacional de doenças nota-se que, tem um fator que está diretamente relacionado a síndrome de Burnout, o código CID:F32 episódio depressivo grave sem sintomas psicóticos. Segundo almeida (2019). O código CID: L50.0 Urticária alérgica é também um dos sintomas físicos que podem aparecer na pessoa acometida pela síndrome.

\section{Conclusão}

Para se construir a pesquisa amparamo-nos em objetivos ligados a conhecer o âmbito escolar e os entraves enfrentados pelos professores para a realização do processo de ensino e aprendizagem como o sistema educacional propõe em 
tempo integral.

Ainda na coleta de dados foi possível perceber apatia dos docentes que alguns não demostraram nenhum interesse em participar da pesquisa, isso porque os sintomas do Burnout já pareciam se apresentar nos mesmos.

Um fator bastante evidenciado no período desta pesquisa foi o tempo para os afazeres pedagógicos dos professores da escola campo de pesquisa, que se apresentavam com sobrecarga e ainda se queixavam por levar trabalho para casa, quando aquele momento que seria em família para repouso, descontração, passeio, viagem, torna-se momentos de excesso de trabalho para o professor que o conduz ao estresse já que usa esse tempo “disponível” para os afazeres das pendências escolares.

Assim, foi possível considerar que a síndrome de Burnout está presente em professores da escola pesquisada. Nesse sentido espera-se que seja possível uma releitura do sistema educacional, que passe por processos de aperfeiçoamento para melhor eficiência e empenho do professor, passando a ser um sistema de educação que veja e reconheça que um professor doente tem que ser tratado.

Nesse olhar, buscar a valorização desse profissional que tanto se esforça para realizar seu trabalho com maestria. Esse trabalho pretende ser ampliado em novas pesquisas para contribuir com o debate da valorização profissional do profissional da educação, o docente.

\section{Considerações Finais}

Diante do exposto, é imprescindível que essa pesquisa seja ampliada a partir de novas contribuições e faz-se necessário uma reflexão acerca das suas falhas ou limitações, a fim de se definir melhorias para que possibilite o alcance de resultados ainda mais significativos no campo das aprendizagens e do desenvolvimento pessoal.

O conhecimento acerca dos fatores preditores da síndrome de burnout facilita a elaboração de medidas e programas de prevenção. Ressalta-se que este estudo apresenta limitações no que se refere ao número de participantes, uma vez que a amostra não foi atingida em razão da não participação de muitos professores na coleta dos dados.

\section{Referências}

Bauer, A., Cassettari, N., \& Oliveira, R. P. D. (2017). Políticas docentes e qualidade da educação: uma revisão da literatura e indicações de política. Ensaio: Avaliação e Políticas Públicas em Educação, 25, 943-970.

Carvalho, M. P., \& Macêdo, M. E. C. (2020). A Síndrome de Burnout em docentes/Burnout Syndrome in Professor's. ID on line Revista de Psicologia, 14(50), 284-301.

da Costa, T. V., \& Scatolin, H. G. (2017). Trabalho e loucura: O adoecimento do sujeito nas organizações. Novas Edições Acadêmicas.

de Borba, R. M. (2020). Qualidade de vida no local de trabalho: o estresse e seus fatores de negatividade. Caderno Profissional de Administração da UNIMEP, 9(1), 76-89.

de Oliveira, Z. R., Maranhão, D., Abbud, I., Zurawski, M. P., Ferreira, M. V., \& Augusto, S. (2020). O trabalho do professor na Educação Infantil. Editora Biruta.

Fabricio, A., Parnoff, G. L., Lopes, L. F. D., \& Brito, L. C. (2020). Síndrome de Burnout: a realidade no ensino docente de uma escola pública de ensino médio da região noroeste/rs. REA-Revista Eletrônica de Administração, 18(2), 250-267.

Freitas, R. N. O. D. (2020). A atividade laboral dos assistentes sociais e o acesso ao adicional de insalubridade.

Jesus, S. D. F., Almeida, N., \& Sgamati, C. A. (2017). A síndrome de Burnout: a causa de doenças em educadores e a sua relação com estresse. Vita et Sanitas, 11(2), 85-93.

Koeppe, C. H. B., Ribeiro, M. E. M., \& Calabró, L. (2020). Por um Ensino investigativo: concepções docentes acerca da pesquisa como atitude e como estratégia pedagógica. Revista Insignare Scientia-RIS, 3(3), 64-83.

Lima, P. A. M., da Silva Pereira, A. R., dos Santos, A. L., \& de Moraes, R. D. (2020). Saúde, Sofrimento, Defesas e Patologias no Trabalho de Professores. Educamazônia-Educação, Sociedade e Meio Ambiente, 25(2, jul-dez), 401-417.

Loiola, E., \& Martins, M. D. C. (2019). Autoeficácia no trabalho e síndrome de burnout em profissionais de enfermagem. Psicologia, Saúde \& Doenças, 20(3), 813-823. 
Research, Society and Development, v. 10, n. 10, e148101018499, 2021

(CC BY 4.0) | ISSN 2525-3409 | DOI: http://dx.doi.org/10.33448/rsd-v10i10.18499

Magalhães, T. A. D., Vieira, M. R. M., Haikal, D. S. A., Nascimento, J. E., Brito, M. F. S. F., Pinho, L., ... \& Silveira, M. F. (2021). Prevalência e fatores associados à síndrome de burnout entre docentes da rede pública de ensino: estudo de base populacional. Revista Brasileira de Saúde Ocupacional, 46.

Mota, Í. D., Farias, G. O., da Silva, R., \& Folle, A. (2017). Síndrome de burnout em estudantes universitários: um olhar sobre as investigações. Motrivivência, $29,243-256$.

Oliveira, F. K. F. F., dos Santos, J. D., Fraga, A. S. B., de Góis, R. M. O., \& da Silva Lima, M. E. (2020). Uso do instrumento maslach burnout inventory como método diagnóstico para síndrome de Burnout em profissionais de enfermagem: uma revisão integrativa. Caderno de Graduação-Ciências Biológicas e da Saúde-UNIT-SERGIPE, 6(1), 189.

Penteado, R. Z., \& Souza, S. D. (2019). Mal-estar, sofrimento e adoecimento do professor: de narrativas do trabalho e da cultura docente à docência como profissão1. Saúde e sociedade, 28, 135-153.

Pertile, G. H., \& Perin, C. (2020). Reflexões sobre a educação no brasil: um olhar histórico sobre o descobrimento da américa e a influência europeia de ensino. Razão e Fé, 22(1).

Pinto, M., Pinto, A., Silva, D. P., Pinto, T., Queiroz, F., \& Melo, S. P. (2015). Síndrome de burnout em docentes. Revista Interdisciplinar, 8(2), $169-177$.

Silva, E. D., \& Ribeiro, W. A. (2020). Síndrome de Burnout e o estresse vivenciados pelos enfermeiros do centro de terapia intensiva: uma revisão de literatura. Research, Society and Development, 9(7), e733974327-e733974327.

Souza, S. T., \& Heinzle, M. R. S. (2020). Metodologias ativas de aprendizagem na educação superior: Aspectos históricos, princípios e propostas de implementação. Revista e-Curriculum, 18(1), 10-39.

Zamoner, Z., Teruya, M. D. C. M., de Souza, M. A., Suave, A. M., \& Monteiro, P. O. (2021). A percepção de professores do ensino fundamental e de alunos da pedagogia sobre o futuro da profissão docente. Humanidades \& Inovação, 8(34), 356-369. 